\title{
Skeletal muscle metabolism on whole-body positron emission tomography during pitching
}

Yasushi Takata ${ }^{1,2}$, Junsuke Nakase ${ }^{1 *}$ (D) Anri Inaki ${ }^{3}$, Takafumi Mochizuki ${ }^{4}$, Kengo Shimozaki ${ }^{1}$, Kazuki Asai ${ }^{1}$, Seigo Kinuya ${ }^{3}$ and Hiroyuki Tsuchiya ${ }^{1}$

\begin{abstract}
Background: Electromyography (EMG) has been used for evaluating skeletal muscle activity during pitching. However, it is difficult to observe the influence of movement on skeletal muscle activity in deep-lying regions of the trunk and extremities using EMG. An alternative method that may be used is the measurement of glucose metabolism of skeletal muscle using positron emission tomography-computed tomography (PET-CT). This technique is a reliable measure of muscle metabolism, demonstrating a high correlation with the intensity of muscle activity. This study aimed to evaluate whole-body skeletal muscle metabolism during pitching using PET-CT.

Methods: Ten uninjured, skilled, adult pitchers, who were active at college or professional level, threw 40 baseballs at maximal effort before an intravenous injection of $37 \mathrm{MBq}$ of ${ }^{18} \mathrm{~F}$-fluorodeoxyglucose (FDG). Subsequently, additional 40 balls were pitched. PET-CT images were obtained 50 min after FDG injection, and regions of interest were defined within 72 muscles. The standardized uptake value (SUV) of FDG by muscle tissue per unit volume was calculated, and the mean SUV of the pitchers was compared with that of a healthy adult control group who did not exercise before the measurements. Statistical analysis was performed using a t-test, and $P<0.05$ was considered statistically significant.
\end{abstract}

Results: Whole-body PET images showed a significant increase in glucose metabolism in the muscle groups of the fingers and toes in both the throwing and non-throwing sides. Additionally, asymmetric increases in glucose metabolism were observed in the muscles of the thigh.

Conclusions: This is the first study to evaluate whole-body muscle metabolism during pitching using PET-CT. Our findings would be useful in determining the training required for pitchers, and can be further applied to other sporting activities that involve throwing.

Keywords: Baseball, Glucose metabolism, Muscle strengthening, Shoulder injuries, Injury prevention, Performance enhancement, Muscles metabolism

\footnotetext{
* Correspondence: nakase1007@yahoo.co.jp

1 Department of Orthopaedic Surgery, Graduate School of Medical Science,

Kanazawa University, 13-1 Takaramachi, Kanazawa 920-0934, Japan

Full list of author information is available at the end of the article
}

(c) The Author(s). 2021 Open Access This article is licensed under a Creative Commons Attribution 4.0 International License, which permits use, sharing, adaptation, distribution and reproduction in any medium or format, as long as you give appropriate credit to the original author(s) and the source, provide a link to the Creative Commons licence, and indicate if changes were made. The images or other third party material in this article are included in the article's Creative Commons licence, unless indicated otherwise in a credit line to the material. If material is not included in the article's Creative Commons licence and your intended use is not permitted by statutory regulation or exceeds the permitted use, you will need to obtain permission directly from the copyright holder. To view a copy of this licence, visit http://creativecommons.org/licenses/by/4.0/ The Creative Commons Public Domain Dedication waiver (http://creativecommons.org/publicdomain/zero/1.0/) applies to the data made available in this article, unless otherwise stated in a credit line to the data. 


\section{Background}

The baseball pitching motion is one of the fastest human motions [1]. Thus, shoulder and elbow pain related to the pitching motion is highly prevalent (approximately $46-57 \%$ of pitchers) [2]. Several studies have been conducted on the prevention of injuries during pitching and the improvement of performance [1-14]. Many of these studies have evaluated motion analysis and skeletal muscle activity using electromyography (EMG) [1, 2, 4$12,14]$. Skeletal muscle activity during pitching has been identified for each body part using EMG [4-12, 14]. Until now, EMG has been the gold standard method in the evaluation of skeletal muscle activity [3]. However, this study focused on positron emission tomography (PET) as a method of evaluating whole-body skeletal muscle metabolism. With PET, it is possible to evaluate whole-body skeletal muscle metabolism in a one-time examination $[15,16]$. Understanding the patterns of whole-body skeletal muscle metabolism in baseball pitching is important not only to define the role of each body part but also to assist in performance enhancement and decrease potential injury [4]. The evidence provided in one study suggests that increased pitching workload may be associated with an increased risk of pain, injury, and arm fatigue in pitchers [17]. Knowledge of the whole-body skeletal muscle metabolism during pitching may facilitate a consideration of other training methods to obtain the necessary muscle strength for pitching. Consequently, it may contribute to reducing overuse injuries and improving performance.

This study aimed to evaluate whole-body skeletal muscle metabolism during a pitching exercise using PET-computed tomography (PET-CT). Based on previous EMG studies and the common occurrence of shoulder injuries among pitchers, it was hypothesized that skeletal muscle metabolism increases in the muscles around the shoulder during pitching.

\section{Methods \\ Participants}

Voluntary applicants were recruited to participate in this study. Those with relevant medical histories, complications, complaints such as pain, and those who did not provide their written consent were excluded. The purpose and potential risks of this study were explained to each participant, who provided written informed consent to participate. The study design was approved by the Ethics Committee of Kanazawa University Hospital (2251-1) and Kanazawa Advanced Medical Center (070209). All human experiments in this study followed the guidelines of the Declaration of Helsinki.

Ten uninjured, skilled, healthy adult male pitchers, who were active at college or professional level with no restriction that would limit maximal effort pitching, participated in this study as the pitcher group. Their physical characteristics (mean \pm standard deviation [SD]) were as follows: age, $21.5 \pm 3.7$ years; height, $175.9 \pm 3.4$ cm; weight, $74.7 \pm 5.2 \mathrm{~kg}$; body mass index, $24.2 \pm 1.8 \mathrm{~kg} /$ $\mathrm{m}^{2}$. Ten healthy adult men who do not exercise and only perform daily life activities served as controls and underwent PET scans to detect the accumulation of ${ }^{18} \mathrm{~F}$-fluorodeoxyglucose (FDG). Their physical characteristics (mean $\pm \mathrm{SD}$ ) were as follows: age, $24.1 \pm 4.1$ years; height, $174.1 \pm 4.9 \mathrm{~cm}$; weight, $78.8 \pm 7.4 \mathrm{~kg}$; body mass index, $25.95 \pm 1.9 \mathrm{~kg} / \mathrm{m}^{2}$. Based on a previous study [15], it was assumed that there would be a two-fold increase in muscle glucose uptake after exercise. None of the participants was on any medication and were all considered healthy based on their medical history and physical examination.

\section{Methodology}

All participants refrained from eating and drinking for at least $6 \mathrm{~h}$ before the FDG-PET assessment, and strenuous physical activity was avoided for at least 1 day before the experiment. The plasma glucose level of each participant was confirmed to be within the normal range (3.89-5.55 $\mathrm{mmol} / \mathrm{l}$ ) before an FDG injection was administered.

After performing sufficient warm-up exercises, the participants threw a total of 40 fast balls to a catcher at maximal effort, on the mound, for $20 \mathrm{~min}$. Subsequently, $37 \mathrm{MBq}$ of FDG was intravenously injected. Additional 40 fast balls were then pitched at maximal effort for another $20 \mathrm{~min}$, followed by $25 \mathrm{~min}$ of rest in a sitting position. According to previous studies $[15,16]$, the influence of exercise is sufficiently reflected by performing the exercise twice (before and after FDG injection). The participants were verbally encouraged to generate maximal effort during pitching. The PET-CT images were obtained $50 \mathrm{~min}$ after FDG injection. The participants were subsequently placed in a supine position on a scanning bed that facilitated longitudinal placement into the gantry of the PET-CT system (Discovery PET/CT 690; GE Healthcare, Milwaukee, WI, USA). Scanning was performed with a $60-\mathrm{cm}$ axial field of view and a transaxial resolution of $6.4 \mathrm{~mm}$ (full-width at half-maximum at the center field of view without a scattering medium). Before emission scanning, an unenhanced CT scan was performed for attenuation correction and anatomical orientation. Furthermore, emission scanning was performed in 3-dimensional (3D) mode $50 \mathrm{~min}$ after FDG administration, and the total emission time ranged from 39 to $42 \mathrm{~min}$. Images were reconstructed using a $3 \mathrm{D}$ ordered-subset expectation-maximization algorithm with two iterations and 16 subsets. After reconstruction, a 6.4-mm FWHM Gaussian post-filter was applied.

Regions of interest (ROI) were manually segmented in 72 skeletal muscles (of the whole-body) from the basal 
neck to the foot. One experienced orthopedic specialist defined all ROIs using plain CT images. The standardized uptake value (SUV) was calculated by overlapping the defined ROI and fusion images. Large vessels were avoided in the course of outlining the muscle areas. The SUV was calculated to quantitatively examine the FDG uptake of the muscle tissue per unit volume according to the equation: $\mathrm{SUV}=\{$ mean ROI count (counts per second $[\mathrm{cps}] /$ pixel $) \times$ calibration factor $(\mathrm{cps} / \mathrm{Bq})\} /$ \{injected dose $(\mathrm{Bq})$ /body weight $(\mathrm{g})$ \}. The ROIs were defined for the right and left sides of the 72 skeletal muscles, and the mean SUV was calculated using the equation below:

mean SUV $=\mathrm{SUV} /$ muscle area

\section{Statistical analyses}

All data are presented as means and SDs. In the determination of the sample size, the $\alpha$ value, power, and effect size of Cohen's d were set at 0.05, 0.95, and 2.0, respectively, and a sample size of $n=10$ was calculated for both the pitcher and control groups. The ShapiroWilk test was performed to ensure a normal distribution. The mean differences of the values were compared using the t-test, and the effect size was presented using Cohen's d. SPSS for Windows ver. 23 (SPSS Inc., Chicago, IL, USA) was used for the analysis. Notably, the minimum significance level was set at $P<0.05$.

\section{Results}

Tables 1 and 2 show the mean SUVs measurements of the pitcher and control groups, and the $95 \%$ confidence intervals, effect sizes, and $P$-values of all muscles. The mean SUVs of the pitcher and control groups were normally distributed, allowing the use of a t-test. In the pitcher group, a significant increase in glucose metabolism was observed in 20 muscles of both the throwing (posterior part of the deltoid $P=0.017$, biceps $P=0.021$, brachialis $P=0.019$, extensor digitorum $P=0.001$, abductor hallucis brevis $P=0.007$, adductor hallucis $P=$ 0.007 , tensor fasciae latae $P=0.02$, biceps femoris $P=$ 0.002 , adductor complex $P=0.029$, popliteus $P=0.01$, anterior tibial $P=0.042$, peroneus longus $P=0.013$, peroneus brevis $P=0.000$, abductor hallucis $P=0.016$, plantar quadrate $P=0.028$, flexor digitorum brevis $P=$ 0.012 , abductor digiti minimi $P=0.014$, flexor hallucis brevis $P=0.001$, abductor hallucis $P=0.017$, interosseous $P=0.005$ ) and non-throwing sides (posterior part of the deltoid $P=0.004$, biceps $P=0.006$, brachialis $P=$ 0.011 , extensor digitorum $P=0.000$, abductor hallucis brevis $P=0.017$, adductor hallucis $P=0.026$, tensor fasciae latae $P=0.049$, biceps femoris $P=0.006$, adductor complex $P=0.017$, popliteus $P=0.014$, anterior tibial
$P=0.038$, peroneus longus $P=0.007$, peroneus brevis $P=0.008$, abductor hallucis $P=0.009$, plantar quadrate $P=0.043$, flexor digitorum brevis $P=0.015$, abductor digiti minimi $P=0.01$, flexor hallucis brevis $P=0.001$, abductor hallucis $P=0.003$, interosseous $P=0.012$ ), 13 muscles of the throwing side (superior part of the trapezius $P=0.026$, horizontal part of trapezius $P=0.024$, teres major $P=0.017$, latissimus dorsi $P=0.045$, brachioradialis $P=0.038$, lumbricalis $P=0.036$, gluteus maximus $P=0.035$, vastus intermedius $P=0.016$, vastus medialis $P=0.011$, semimembranosus $P=0.029$, extensor hallucis longus $P=0.022$, extensor digitorum longus $P=0.031$, flexor hallucis longus $P=0.041$ ) and 8 muscles of the non-throwing side (levator scapulae $P=0.049$, forearm flexors $P=0.015$, iliacus $P=0.005$, gluteus minimus $P=0.039$, sartorius $P=0.005$, gracilis $P=0.005$, semitendinosus $P=0.008$, triceps surae $P=0.035$ ). In summary, the results of the pitchers'whole-body PET images (Fig. 1) revealed significant increases in glucose metabolism in the muscle groups of the fingers and toes. In addition, in the lower limbs, asymmetric increases in glucose metabolism were observed in the thigh muscles. However, the rotator cuff or the trunk muscles did not exhibit increased glucose metabolism.

\section{Discussion}

In this study, the whole-body skeletal muscle metabolism during pitching motion was evaluated using PET. An accumulation of FDG was observed in the relatively small skeletal muscles of the fingers and toes, and asymmetric accumulation was observed in the thigh muscles. Interestingly, there was minimal accumulation of FDG in the rotator cuff and trunk muscles, which are considered important for the throwing motion.

EMG has been used in the evaluation of skeletal muscle activity during pitching [1, 6-13]. Since EMG has excellent time resolution, it was useful for investigating skeletal muscle activity in each throwing phase by synchronizing the time axis to motion analysis [4]. However, when using EMG with surface electrodes, since it is necessary to attach the electrode beforehand to the muscle to be studied, it is only possible to measure the muscle that can be touched from the body surface and the muscles scheduled for investigation [4-6]. Therefore, it is difficult to observe the influence of exercise on skeletal muscle activity in deep-lying regions of the trunk and extremities [16]. Alternatively, wire electrodes can be used for deeper skeletal muscles; however, they are invasive $[8,12]$. In addition, the accuracy of the insertion site and the influence of noise artifacts similarly have a significant impact $[8,12]$. Furthermore, in evaluation using EMG, the skeletal muscles of the whole-body are invisible, and the device's cords and electrodes interfere with motion [16]. Hence, it becomes impossible to study 
Table 1 SUVs for the upper extremity skeletal muscles between the pitcher and control groups

\begin{tabular}{|c|c|c|c|c|c|c|c|c|c|c|}
\hline \multirow[t]{3}{*}{ Muscles } & \multicolumn{5}{|c|}{ Throwing side } & \multicolumn{5}{|c|}{ Non-throwing side } \\
\hline & \multicolumn{2}{|l|}{ SUV value } & \multirow{2}{*}{$\begin{array}{l}\text { Confidence } \\
\text { interval }\end{array}$} & \multirow{2}{*}{$\begin{array}{l}\text { Effect } \\
\text { size }\end{array}$} & \multirow{2}{*}{$\begin{array}{l}P \text { - } \\
\text { value }\end{array}$} & \multicolumn{2}{|c|}{ SUV value } & \multirow{2}{*}{$\begin{array}{l}\text { Confidence } \\
\text { interval }\end{array}$} & \multirow{2}{*}{$\begin{array}{l}\text { Effect } \\
\text { size }\end{array}$} & \multirow{2}{*}{$\begin{array}{l}P \text { - } \\
\text { value }\end{array}$} \\
\hline & Pitching & Control & & & & Pitching & Control & & & \\
\hline Superior part of trapezius & $0.70 \pm 0.14$ & $0.58 \pm 0.08$ & {$[0.02,0.24]$} & 1.09 & $0.026^{a}$ & $0.64 \pm 0.06$ & $0.57 \pm 0.09$ & {$[-0.01,0.14]$} & 0.85 & 0.074 \\
\hline Horizontal part of trapezius & $0.74 \pm 0.09$ & $0.65 \pm 0.08$ & {$[0.01,0.17]$} & 1.11 & $0.024^{\mathrm{a}}$ & $0.74 \pm 0.14$ & $0.67 \pm 0.08$ & {$[-0.03,0.18]$} & 0.67 & 0.153 \\
\hline Inferior part of trapezius & $0.70 \pm 0.07$ & $0.73 \pm 0.08$ & {$[-0.09,0.05]$} & 0.33 & 0.468 & $0.74 \pm 0.13$ & $0.69 \pm 0.07$ & {$[-0.05,0.15]$} & 0.45 & 0.325 \\
\hline Levator scapulae & $0.71 \pm 0.18$ & $0.58 \pm 0.10$ & {$[-0.01,0.27]$} & 0.88 & 0.07 & $0.71 \pm 0.15$ & $0.58 \pm 0.13$ & {$[0.00,0.26]$} & 0.94 & $0.049^{\mathrm{a}}$ \\
\hline Rhomboids & $0.65 \pm 0.08$ & $0.68 \pm 0.11$ & {$[-0.11,0.06]$} & 0.27 & 0.554 & $0.67 \pm 0.13$ & $0.76 \pm 0.09$ & {$[-0.19,0.02]$} & 0.76 & 0.106 \\
\hline Serratus anterior & $0.76 \pm 0.07$ & $0.71 \pm 0.08$ & {$[-0.06,0.16]$} & 0.46 & 0.316 & $0.74 \pm 0.11$ & $0.68 \pm 0.10$ & {$[-0.04,0.15]$} & 0.56 & 0.23 \\
\hline Pectoralis minor & $0.72 \pm 0.09$ & $0.62 \pm 0.12$ & {$[-0.01,0.19]$} & 0.89 & 0.062 & $0.70 \pm 0.14$ & $0.68 \pm 0.10$ & {$[-0.09,0.14]$} & 0.21 & 0.649 \\
\hline Subscapularis & $0.76 \pm 0.07$ & $0.79 \pm 0.08$ & {$[-0.10,0.05]$} & 0.32 & 0.481 & $0.80 \pm 0.09$ & $0.77 \pm 0.09$ & {$[-0.06,0.12]$} & 0.3 & 0.509 \\
\hline Supraspinatus & $0.75 \pm 0.10$ & $0.72 \pm 0.14$ & {$[-0.08,0.14]$} & 0.25 & 0.579 & $0.76 \pm 0.07$ & $0.79 \pm 0.09$ & {$[-0.10,0.05]$} & 0.33 & 0.465 \\
\hline Infraspinatus & $0.81 \pm 0.07$ & $0.83 \pm 0.09$ & {$[-0.10,0.06]$} & 0.22 & 0.626 & $0.86 \pm 0.11$ & $0.80 \pm 0.11$ & {$[-0.04,0.17]$} & 0.56 & 0.227 \\
\hline Teres minor & $0.88 \pm 0.10$ & $0.88 \pm 0.19$ & {$[-0.14,0.15]$} & 0.05 & 0.906 & $0.88 \pm 0.10$ & $0.85 \pm 0.14$ & {$[-0.09,0.15]$} & 0.25 & 0.589 \\
\hline Teres major & $0.85 \pm 0.22$ & $0.63 \pm 0.14$ & {$[0.04,0.39]$} & 1.17 & $0.017^{\mathrm{a}}$ & $0.93 \pm 0.49$ & $0.65 \pm 0.16$ & {$[-0.08,0.64]$} & 0.77 & 0.113 \\
\hline Anterior part of deltoid & $0.60 \pm 0.20$ & $0.62 \pm 0.12$ & {$[-0.18,0.13]$} & 0.14 & 0.758 & $0.68 \pm 0.37$ & $0.60 \pm 0.10$ & {$[-0.18,0.34]$} & 0.3 & 0.515 \\
\hline Middle part of deltoid & $0.66 \pm 0.16$ & $0.64 \pm 0.11$ & {$[-0.11,0.15]$} & 0.12 & 0.787 & $0.66 \pm 0.13$ & $0.64 \pm 0.09$ & {$[-0.09,0.13]$} & 0.18 & 0.69 \\
\hline Posterior part of deltoid & $0.77 \pm 0.10$ & $0.65 \pm 0.06$ & {$[0.04,0.20]$} & 1.46 & $0.017^{\mathrm{a}}$ & $0.78 \pm 0.11$ & $0.63 \pm 0.10$ & {$[0.06,0.25]$} & 1.49 & $0.004^{\mathrm{a}}$ \\
\hline Latissimus dorsi & $0.76 \pm 0.29$ & $0.55 \pm 0.09$ & {$[0.01,0.43]$} & 1.02 & $0.045^{\mathrm{a}}$ & $0.90 \pm 0.49$ & $0.57 \pm 0.09$ & {$[-0.02,0.68]$} & 0.94 & 0.064 \\
\hline Pectoralis major & $0.71 \pm 0.22$ & $0.56 \pm 0.12$ & {$[-0.01,0.32]$} & 0.87 & 0.067 & $0.68 \pm 0.37$ & $0.58 \pm 0.11$ & {$[-0.05,0.26]$} & 0.64 & 0.168 \\
\hline Coracobrachialis & $0.97 \pm 0.13$ & $0.90 \pm 0.12$ & {$[-0.05,0.19]$} & 0.56 & 0.229 & $0.66 \pm 0.13$ & $0.88 \pm 0.14$ & {$[-0.15,0.11]$} & 0.13 & 0.769 \\
\hline Biceps & $0.85 \pm 0.33$ & $0.57 \pm 0.11$ & {$[0.05,0.51]$} & 1.13 & $0.021^{a}$ & $1.00 \pm 0.36$ & $0.59 \pm 0.12$ & {$[0.15,0.68]$} & 1.54 & $0.006^{\mathrm{a}}$ \\
\hline Brachialis & $0.90 \pm 0.23$ & $0.67 \pm 0.16$ & {$[0.04,0.42]$} & 1.16 & $0.019^{a}$ & $0.95 \pm 0.31$ & $0.63 \pm 0.14$ & {$[0.09,0.55]$} & 1.32 & $0.011^{\mathrm{a}}$ \\
\hline Triceps & $0.74 \pm 0.36$ & $0.55 \pm 0.10$ & {$[-0.07,0.45]$} & 0.72 & 0.137 & $0.60 \pm 0.31$ & $0.54 \pm 0.12$ & {$[-0.15,0.29]$} & 0.29 & 0.519 \\
\hline Anconeus & $0.77 \pm 0.17$ & $0.78 \pm 0.14$ & {$[-0.15,0.14]$} & 0.04 & 0.923 & $0.84 \pm 0.21$ & $0.74 \pm 0.18$ & {$[-0.08,0.29]$} & 0.53 & 0.25 \\
\hline Pronator teres & $0.77 \pm 0.26$ & $0.71 \pm 0.19$ & {$[-0.15,0.27]$} & 0.26 & 0.562 & $0.82 \pm 0.36$ & $0.76 \pm 0.18$ & {$[-0.20,0.33]$} & 0.22 & 0.631 \\
\hline Forearm flexors & $0.96 \pm 0.40$ & $0.76 \pm 0.08$ & {$[-0.08,0.49]$} & 0.71 & 0.144 & $1.04 \pm 0.32$ & $0.73 \pm 0.10$ & {$[0.07,0.54]$} & 1.29 & $0.015^{\mathrm{a}}$ \\
\hline Pronator quadratus & $0.87 \pm 0.53$ & $0.72 \pm 0.19$ & {$[-0.25,0.53]$} & 0.36 & 0.432 & $0.72 \pm 0.16$ & $0.72 \pm 0.19$ & {$[-0.16,0.17]$} & 0.02 & 0.971 \\
\hline Brachioradialis & $0.87 \pm 0.30$ & $0.64 \pm 0.13$ & {$[0.01,0.44]$} & 1.00 & $0.038^{\mathrm{a}}$ & $0.71 \pm 0.28$ & $0.54 \pm 0.09$ & {$[-0.04,0.37]$} & 0.8 & 0.102 \\
\hline Extensor digitorum & $1.67 \pm 0.49$ & $0.81 \pm 0.15$ & {$[0.35,1.09]$} & 1.90 & $0.001^{\mathrm{a}}$ & $1.96 \pm 0.64$ & $0.88 \pm 0.17$ & {$[0.64,1.51]$} & 2.31 & $0.000^{\mathrm{a}}$ \\
\hline Abductor hallucis brevis & $1.67 \pm 0.71$ & $0.88 \pm 0.30$ & {$[0.26,1.32]$} & 1.45 & $0.007^{\mathrm{a}}$ & $1.30 \pm 0.57$ & $0.77 \pm 0.14$ & {$[0.11,0.94]$} & 1.27 & $0.017^{\mathrm{a}}$ \\
\hline Adductor hallucis & $2.10 \pm 0.88$ & $1.13 \pm 0.27$ & {$[0.33,1.62]$} & 1.50 & $0.007^{\mathrm{a}}$ & $1.51 \pm 0.54$ & $1.03 \pm 0.24$ & {$[0.07,0.88]$} & 1.13 & $0.026^{\mathrm{a}}$ \\
\hline Abductor digiti minimi pedis & $1.06 \pm 0.64$ & $0.94 \pm 0.23$ & {$[-0.32,0.57]$} & 0.26 & 0.569 & $0.97 \pm 0.29$ & $0.86 \pm 0.20$ & {$[-0.13,0.35]$} & 0.44 & 0.341 \\
\hline Lumbricalis & $1.64 \pm 0.77$ & $1.02 \pm 0.41$ & {$[0.05,1.21]$} & 1.02 & $0.036^{\mathrm{a}}$ & $1.36 \pm 0.67$ & $0.97 \pm 0.21$ & {$[-0.08,0.85]$} & 0.78 & 0.097 \\
\hline
\end{tabular}

${ }^{a}$ Statistically significant

SUV Standardized uptake value

Values are presented as mean \pm standard deviation

muscle activity in conditions that actually replicate the real-world pitching environment and performance [16]. Therefore, only partial investigations on the upper limbs $[6-9,11,12]$, lower limbs $[4,10,13]$, or the trunk [14] have been performed in pitchers so far.

Some studies have utilized FDG-PET to display cumulative muscle metabolism during exercise $[15,16,18-$ 20]. FDG taken up by muscle cells is not metabolized and remains in the cells as FDG-6-phosphate after phosphorylation [15]. Thus, FDG accumulation in the muscle can be used as a parameter of glucose uptake by the muscle and is an indicator of muscle activity [15]. Glucose metabolism measured by FDG-PET demonstrates a high correlation with the intensity of muscle activity, and its reliability as an index for measuring muscle activity has been confirmed [20]. Fujimoto et al. [19] and Tashiro et al. [21] used PET in the evaluation of muscle metabolism during running (one of the first studies). Other studies have investigated tissue glucose uptake with PET during tasks such as isometric muscle 
Table 2 SUVs for the trunk and lower extremity skeletal muscles between the pitcher and control groups

\begin{tabular}{|c|c|c|c|c|c|c|c|c|c|c|}
\hline \multirow[t]{3}{*}{ Muscles } & \multicolumn{5}{|c|}{ Throwing side } & \multicolumn{5}{|c|}{ Non-throwing side } \\
\hline & \multicolumn{2}{|l|}{ SUV value } & \multirow{2}{*}{$\begin{array}{l}\text { Confidence } \\
\text { interval }\end{array}$} & \multirow{2}{*}{$\begin{array}{l}\text { Effect } \\
\text { size }\end{array}$} & \multirow{2}{*}{$\begin{array}{l}P \text { - } \\
\text { value }\end{array}$} & \multicolumn{2}{|l|}{ SUV value } & \multirow{2}{*}{$\begin{array}{l}\text { Confidence } \\
\text { interval }\end{array}$} & \multirow{2}{*}{$\begin{array}{l}\text { Effect } \\
\text { size }\end{array}$} & \multirow{2}{*}{$\begin{array}{l}P \text { - } \\
\text { value }\end{array}$} \\
\hline & Pitching & Control & & & & Pitching & Control & & & \\
\hline Abdominal rectus & $0.52 \pm 0.09$ & $0.57 \pm 0.13$ & {$[-0.16,0.05]$} & 0.66 & 0.313 & $0.50 \pm 0.09$ & $0.53 \pm 0.11$ & {$[-0.12,0.07]$} & 0.6 & 0.591 \\
\hline Abdominal external oblique & $0.50 \pm 0.05$ & $0.46 \pm 0.07$ & {$[-0.01,0.10]$} & 0.4 & 0.13 & $0.51 \pm 0.07$ & $0.48 \pm 0.06$ & {$[-0.04,0.09]$} & 0.45 & 0.371 \\
\hline Abdominal internal oblique & $0.64 \pm 0.09$ & $0.62 \pm 0.09$ & {$[-0.07,0.10]$} & 0.07 & 0.676 & $0.67 \pm 0.11$ & $0.64 \pm 0.10$ & {$[-0.07,0.13]$} & 0.88 & 0.572 \\
\hline Transverse abdominal & $0.69 \pm 0.12$ & $0.69 \pm 0.13$ & {$[-0.11,0.11]$} & 0.22 & 0.978 & $0.72 \pm 0.17$ & $0.60 \pm 0.13$ & {$[-0.02,0.26]$} & 1.21 & 0.095 \\
\hline Greater psoas & $0.85 \pm 0.18$ & $0.73 \pm 0.13$ & {$[-0.08,0.21]$} & 0.37 & 0.359 & $0.91 \pm 0.22$ & $0.87 \pm 0.12$ & {$[-0.13,0.20]$} & 0.38 & 0.622 \\
\hline Lumbar quadrate & $0.66 \pm 0.08$ & $0.64 \pm 0.14$ & {$[-0.08,0.13]$} & 0.52 & 0.641 & $0.68 \pm 0.09$ & $0.60 \pm 0.11$ & {$[-0.01,0.17]$} & 0.52 & 0.089 \\
\hline Erector spinae & $0.78 \pm 0.13$ & $0.76 \pm 0.12$ & {$[-0.10,0.13]$} & 0.16 & 0.743 & $0.79 \pm 0.10$ & $0.74 \pm 0.10$ & {$[-0.05,0.14]$} & 0.1 & 0.299 \\
\hline \|liacus & $0.92 \pm 0.10$ & $0.98 \pm 0.23$ & {$[-0.23,0.11]$} & 0.88 & 0.467 & $1.37 \pm 0.36$ & $0.95 \pm 0.14$ & {$[0.16,0.69]$} & 1.52 & $0.005^{\mathrm{a}}$ \\
\hline Gluteus maximus & $0.75 \pm 0.15$ & $0.64 \pm 0.06$ & {$[0.01,0.22]$} & 1.02 & $0.035^{\mathrm{a}}$ & $0.80 \pm 0.19$ & $0.68 \pm 0.10$ & {$[-0.03,0.26]$} & 1.05 & 0.1 \\
\hline Gluteus medius & $0.76 \pm 0.08$ & $0.73 \pm 0.10$ & {$[-0.06,0.11]$} & 0.17 & 0.566 & $0.86 \pm 0.18$ & $0.75 \pm 0.05$ & {$[-0.02,0.25]$} & 0.73 & 0.092 \\
\hline Gluteus minimus & $1.17 \pm 0.57$ & $0.98 \pm 0.13$ & {$[-0.20,0.58]$} & 0.47 & 0.33 & $1.68 \pm 1.00$ & $0.91 \pm 0.18$ & {$[0.05,1.49]$} & 0.9 & $0.039^{a}$ \\
\hline Piriformis & $0.93 \pm 0.16$ & $0.93 \pm 0.16$ & {$[-0.15,0.15]$} & 0.59 & 0.977 & $1.11 \pm 0.27$ & $0.97 \pm 0.19$ & {$[-0.09,0.35]$} & 0.05 & 0.22 \\
\hline Internal obturator & $0.93 \pm 0.19$ & $1.20 \pm 0.37$ & {$[-0.54,0.02]$} & 1.45 & 0.062 & $0.99 \pm 0.21$ & $1.04 \pm 0.37$ & {$[-0.34,0.23]$} & 0.5 & 0.712 \\
\hline Obturator externus & $0.95 \pm 0.17$ & $1.00 \pm 0.24$ & {$[-0.24,0.16]$} & 0.86 & 0.677 & $1.12 \pm 0.33$ & $1.02 \pm 0.19$ & {$[-0.15,0.35]$} & 0.12 & 0.414 \\
\hline Tensor fasciae latae & $0.60 \pm 0.19$ & $0.43 \pm 0.08$ & {$[0.03,0.31]$} & 1.15 & $0.020^{a}$ & $0.89 \pm 0.61$ & $0.45 \pm 0.05$ & {$[0.00,0.88]$} & 0.94 & $0.049^{a}$ \\
\hline Rectus femoris & $0.67 \pm 0.36$ & $0.49 \pm 0.08$ & {$[-0.06,0.43]$} & 0.67 & 0.13 & $0.90 \pm 0.63$ & $0.49 \pm 0.08$ & {$[-0.05,0.86]$} & 0.78 & 0.074 \\
\hline Vastus lateralis & $0.64 \pm 0.12$ & $0.55 \pm 0.10$ & {$[-0.01,0.19]$} & 0.66 & 0.088 & $0.60 \pm 0.15$ & $0.55 \pm 0.10$ & {$[-0.08,0.17]$} & 0.42 & 0.424 \\
\hline vastus intermedius & $0.80 \pm 0.12$ & $0.64 \pm 0.15$ & {$[0.03,0.29]$} & 1.51 & $0.016^{\mathrm{a}}$ & $0.76 \pm 0.11$ & $0.69 \pm 0.10$ & {$[-0.03,0.17]$} & 0.85 & 0.15 \\
\hline vastus medialis & $0.68 \pm 0.13$ & $0.55 \pm 0.06$ & {$[0.03,0.23]$} & 1.21 & $0.011^{\mathrm{a}}$ & $0.62 \pm 0.14$ & $0.55 \pm 0.06$ & {$[-0.03,0.17]$} & 0.73 & 0.14 \\
\hline Sartorius & $0.66 \pm 0.31$ & $0.54 \pm 0.10$ & {$[-0.10,0.34]$} & 0.43 & 0.264 & $0.92 \pm 0.34$ & $0.52 \pm 0.08$ & {$[0.15,0.64]$} & 1.4 & $0.005^{\mathrm{a}}$ \\
\hline Gracilis & $0.58 \pm 0.20$ & $0.43 \pm 0.10$ & {$[0.00,0.30]$} & 0.58 & 0.052 & $0.59 \pm 0.16$ & $0.40 \pm 0.10$ & {$[0.07,0.31]$} & 0.98 & $0.005^{\mathrm{a}}$ \\
\hline Semimembranosus & $0.78 \pm 0.32$ & $0.52 \pm 0.07$ & {$[0.03,0.50]$} & 0.92 & $0.029^{a}$ & $0.70 \pm 0.21$ & $0.55 \pm 0.07$ & {$[0.00,0.29]$} & 0.84 & 0.051 \\
\hline Semitendinosus & $0.88 \pm 0.59$ & $0.46 \pm 0.10$ & {$[0.00,0.84]$} & 0.85 & 0.051 & $0.64 \pm 0.15$ & $0.48 \pm 0.08$ & {$[0.05,0.27]$} & 1.44 & $0.008^{\mathrm{a}}$ \\
\hline Biceps femoris & $0.67 \pm 0.11$ & $0.53 \pm 0.06$ & {$[0.06,0.23]$} & 1.77 & $0.002^{\mathrm{a}}$ & $0.68 \pm 0.12$ & $0.54 \pm 0.08$ & {$[0.05,0.24]$} & 1.59 & $0.006^{a}$ \\
\hline Adductor complex & $0.74 \pm 0.09$ & $0.66 \pm 0.07$ & {$[0.01,0.16]$} & 1.24 & $0.029^{a}$ & $0.75 \pm 0.08$ & $0.65 \pm 0.09$ & {$[0.02,0.17]$} & 1.85 & $0.017^{\mathrm{a}}$ \\
\hline Popliteus & $0.98 \pm 0.19$ & $0.77 \pm 0.11$ & {$[0.06,0.36]$} & 1.18 & $0.010^{\mathrm{a}}$ & $0.97 \pm 0.17$ & $0.80 \pm 0.12$ & {$[0.04,0.32]$} & 0.86 & $0.014^{\mathrm{a}}$ \\
\hline Anterior tibial & $1.18 \pm 0.50$ & $0.79 \pm 0.17$ & {$[0.02,0.76]$} & 0.89 & $0.042^{\mathrm{a}}$ & $1.28 \pm 0.68$ & $0.75 \pm 0.20$ & {$[0.03,1.03]$} & 0.93 & $0.038^{\mathrm{a}}$ \\
\hline Extensor hallucis longus & $1.11 \pm 0.32$ & $0.81 \pm 0.19$ & {$[0.05,0.55]$} & 1.05 & $0.022^{a}$ & $1.07 \pm 0.33$ & $0.88 \pm 0.23$ & {$[-0.07,0.46]$} & 0.58 & 0.143 \\
\hline Extensor digitorum longus & $0.98 \pm 0.24$ & $0.77 \pm 0.13$ & {$[0.02,0.39]$} & 1.01 & $0.031^{\mathrm{a}}$ & $1.06 \pm 0.31$ & $0.82 \pm 0.17$ & {$[0.00,0.47]$} & 1.23 & 0.051 \\
\hline Peroneus longus & $1.03 \pm 0.39$ & $0.66 \pm 0.09$ & {$[0.10,0.66]$} & 1.23 & $0.013^{\mathrm{a}}$ & $1.07 \pm 0.38$ & $0.67 \pm 0.15$ & {$[0.12,0.69]$} & 1.09 & $0.007^{\mathrm{a}}$ \\
\hline Peroneus brevis & $1.26 \pm 0.39$ & $0.61 \pm 0.08$ & {$[0.37,0.93]$} & 1.92 & $0.000^{\mathrm{a}}$ & $1.63 \pm 0.99$ & $0.57 \pm 0.14$ & {$[0.34,1.77]$} & 1.17 & $0.008^{\mathrm{a}}$ \\
\hline Flexor digitorum longus & $0.97 \pm 0.19$ & $0.82 \pm 0.40$ & {$[-0.14,0.45]$} & 0.11 & 0.29 & $0.92 \pm 0.24$ & $0.75 \pm 0.25$ & {$[-0.06,0.39]$} & 0.53 & 0.143 \\
\hline Posterior tibial & $1.06 \pm 0.32$ & $0.81 \pm 0.22$ & {$[-0.01,0.51]$} & 0.42 & 0.055 & $0.85 \pm 0.14$ & $0.83 \pm 0.19$ & {$[-0.14,0.18]$} & 0.44 & 0.776 \\
\hline Flexor hallucis longus & $1.10 \pm 0.36$ & $0.74 \pm 0.39$ & {$[0.02,0.72]$} & 0.19 & $0.041^{\mathrm{a}}$ & $1.06 \pm 0.29$ & $0.78 \pm 0.34$ & {$[-0.01,0.58]$} & 0.06 & 0.057 \\
\hline Triceps surae & $1.23 \pm 0.58$ & $0.80 \pm 0.43$ & {$[-0.06,0.91]$} & 0.32 & 0.079 & $1.16 \pm 0.54$ & $0.73 \pm 0.25$ & {$[0.03,0.83]$} & 0.54 & $0.035^{\mathrm{a}}$ \\
\hline Abductor hallucis & $1.99 \pm 1.33$ & $0.74 \pm 0.24$ & {$[0.29,2.20]$} & 1.01 & $0.016^{\mathrm{a}}$ & $1.30 \pm 0.50$ & $0.74 \pm 0.34$ & {$[0.16,0.96]$} & 0.97 & $0.009^{a}$ \\
\hline Plantar quadrate & $1.19 \pm 0.52$ & $0.77 \pm 0.16$ & {$[0.05,0.78]$} & 0.67 & $0.028^{\mathrm{a}}$ & $1.16 \pm 0.44$ & $0.82 \pm 0.23$ & {$[0.01,0.68]$} & 0.54 & $0.043^{\mathrm{a}}$ \\
\hline Flexor digitorum brevis & $1.55 \pm 0.76$ & $0.79 \pm 0.21$ & {$[0.21,1.31]$} & 1.06 & $0.012^{a}$ & $1.44 \pm 0.76$ & $0.71 \pm 0.22$ & {$[0.18,1.28]$} & 1 & $0.015^{\mathrm{a}}$ \\
\hline Abductor digiti minimi & $1.13 \pm 0.44$ & $0.69 \pm 0.27$ & {$[0.10,0.79]$} & 1.08 & $0.014^{\mathrm{a}}$ & $1.34 \pm 0.68$ & $0.69 \pm 0.24$ & {$[0.18,1.13]$} & 0.91 & $0.010^{\mathrm{a}}$ \\
\hline Flexor hallucis brevis & $1.75 \pm 0.68$ & $0.77 \pm 0.26$ & {$[0.47,1.49]$} & 1.71 & $0.001^{a}$ & $1.81 \pm 0.66$ & $0.81 \pm 0.31$ & {$[0.50,1.51]$} & 1.65 & $0.001^{a}$ \\
\hline Abductor hallucis & $1.65 \pm 0.91$ & $0.80 \pm 0.24$ & {$[0.19,1.51]$} & 1.09 & $0.017^{\mathrm{a}}$ & $1.58 \pm 0.54$ & $0.87 \pm 0.33$ & {$[0.28,1.12]$} & 1.43 & $0.003^{\mathrm{a}}$ \\
\hline Interosseous & $1.37 \pm 0.59$ & $0.68 \pm 0.22$ & {$[0.25,1.12]$} & 1.38 & $0.005^{\mathrm{a}}$ & $1.21 \pm 0.49$ & $0.71 \pm 0.23$ & {$[0.13,0.88]$} & 1.3 & $0.012^{\mathrm{a}}$ \\
\hline
\end{tabular}




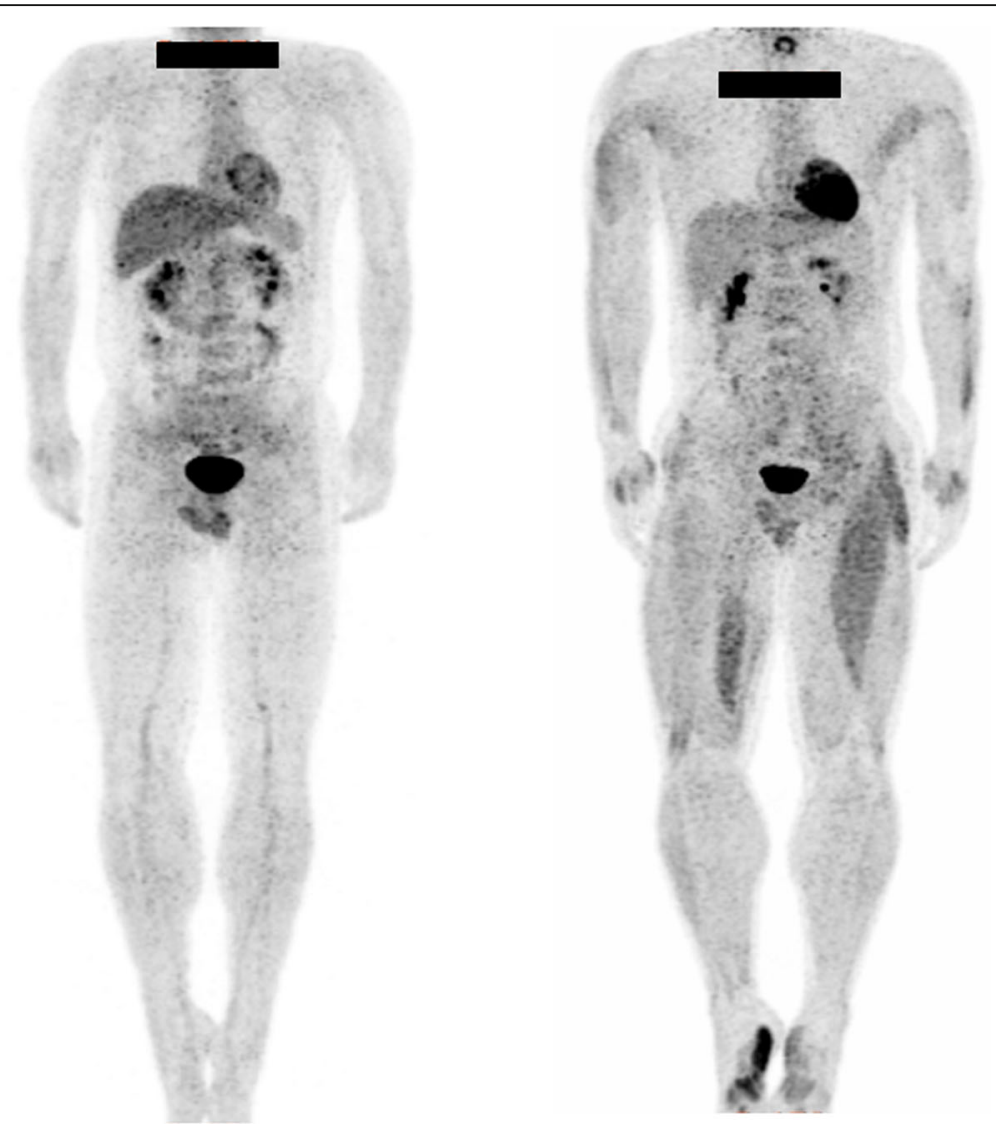

Fig. 1 Representative whole-body positron emission tomography images of the control group (left side) and the pitcher group (right side)

contractions [15] and dynamic strength exercises [22], and during more complex tasks requiring endurance, such as walking [23], running [24], and double poling [25]. In a previous study, whole-body PET-CT was used to examine where glucose uptake occurs in the skeletal muscles in a single performance of Part 2 of the Fédération Internationale de Football Association's 11+ injury prevention program [15] and following its 4-week routine performance [16]. Bojsen-Møller et al. [25] proposed that PET imaging might be a promising adjunct modality or an alternative to more traditional methods of investigating muscle activity during complex human movements. Rudroff et al. [22] mentioned that PET has the potential of being the "gold standard" of metabolic imaging for exercise physiology.

In this study, it was observed that the accumulation of FDG in the relatively small skeletal muscles of the fingers, especially in the muscles that have their origin and insertion within the hand (intrinsic muscles). The fingers control, which is the end of the kinetic chain of the pitching motion, is extremely precise and important [26]. The intrinsic muscles are known to play a vital role in the flexing and stabilization of the metacarpophalangeal (MCP) joint. Ketchum et al. [27] reported that the intrinsic muscles contribute more than $70 \%$ of the overall moment of the flexion at the MCP joint. However, studies on the intrinsic finger muscles activity during pitching have not been conducted. This is presumably because the evaluation of intrinsic muscles is difficult with EMG. Using another approach, Kinoshita et al. [28] measured finger force for fastball pitching; a linear relationship was observed between peak forces and ball velocity, and the peak ball reaction force for fastballs exceeded $80 \%$ of maximum finger strength. According to these studies, the training of finger muscles is important for enhancing pitching performance [26-28]. Similarly, FDG accumulation increased in multiple intrinsic foot muscles in this study. The control of the intrinsic foot muscles is equally important for the first step in the kinetic chain of the pitching motion [29]. However, the intrinsic foot muscles activity has not attracted significant attention until now due to the difficulty to be evaluated with EMG $[4,10]$.

Some skeletal muscles of the femoral region demonstrated unilateral accumulation of FDG. This might reflect the asymmetric movements performed during pitching. The pitching motion has been classified into six phases [30]. Specifically, it was suggested that the iliacus on the non-throwing side is involved in the lifting 
movement of the lower limbs during the wind-up phase $[4,10,13,29,30]$. On the contrary, the medial hamstrings, vastus medialis, and adductor muscle on the throwing side are responsible for the translational motion during the stride phase to the acceleration phase and the raising motion of the lower extremity during the deceleration phase $[4,10,13,29,30]$. The sartorius and gracilis muscles on the non-throwing side are involved in the process of standing up on one leg during the acceleration to follow-through phase $[4,10,13,29,30]$. In a previous EMG study, Yamanouchi [10] investigated the abductor, adductor, quadriceps, biceps, tibialis anterior, and gastrocnemius muscles activities; it was concluded that the abductor and adductor muscles play important roles. Similarly, activation of the adductor muscle was observed in this study. In the investigation of the vastus medialis, biceps femoris, rectus femoris, gluteus maximus, and gastrocnemius muscles activities by Campbell et al. [4], it was concluded that stride foot contact on ball release was the most demanding phase of the pitching motion with a very high bilateral muscular activation. The iliacus and sartorius muscles were excluded from the investigation [4]. Since the iliac muscle is in a deep position, it would be difficult to investigate its activity using EMG. Regarding the biceps femoris muscle on the throwing side, reports equally indicated a high activity [4, 10, 13]. In addition, Erickson et al. [13] conducted EMG studies and reported a higher hamstring activity in the throwing side than the nonthrowing side, which is consistent with this study.

Interestingly, minimal accumulation of FDG was observed in the rotator cuff and trunk muscles in this study, suggesting the possibility that these muscles contribute less to the throwing movement. The shoulder is an integral part of the kinetic chain in the throwing motion. Rotator cuff muscles act as dynamic stabilizers of the glenohumeral joint during throwing [6,7]. An initial report of shoulder muscle activity during baseball pitching evaluated by EMG was published in 1983 by Jobe et al. [11]. Since then, considerable research using EMG for the muscles around the shoulder joint during pitching has been performed $[6-9,11,12]$. According to these studies, the rotator cuff demonstrated high activity during the early cocking to deceleration phases $[6-9,11,12]$. This study showed no increase in glucose metabolism in the rotator cuffs. Around the shoulder joint, increased metabolism was observed in the posterior aspect of the deltoid muscles on both sides, the trapezius muscle, teres major, latissimus dorsi on the pitching side, and the levator scapulae on the nonthrowing side in this study. Similarly, increased metabolism in both sides of the biceps and brachial muscles was observed in this study.
There is only one report on muscle activity of the trunk during pitching measured by EMG [14]. In the investigation of the muscle activity of the abdominal rectus, abdominal oblique, lumbar paraspinous, and gluteus maximus muscles by Watkins et al. [14], it was reported that the abdominal rectus and abdominal oblique muscles on the non-throwing side demonstrated an increase in the activity level. In this study, no increase in glucose metabolism was observed in the trunk muscles; however, increased metabolism was observed only in the gluteus maximus muscle on the throwing side.

Understanding the patterns of whole-body muscle metabolism in baseball pitching is important to enhance performance and decrease injury potential [4]. For practical application, data from this study provide strength and conditioning professionals more definitive evidence on the importance of finger and foot muscular strengths and endurance training for pitchers. Furthermore, training regimens promoting both bilateral and unilateral lower extremity muscular strengths and endurance in multiple planes (similar to the movements in pitching) are critical to address the specific demands of the pitching motion $[1,3,4,30,31]$.

This study had some limitations. First, the number of throws that is optimal for measuring FDG accumulation is unclear. Based on previous studies, it was better to exercise for $20 \mathrm{~min}$ before and after the FDG injection. Thus, the participants threw 40 balls in 20 min before and after FDG injection. However, since the pitching motion was completed in approximately $2 \mathrm{~s}$, the appropriateness of the exercise load in this study is questionable. In addition, it is possible that the motion and the pattern of muscle metabolism changed with fatigue [13]. We are planning to examine these issues by increasing the number of throws in future studies. Second, the method of FDG-PET only accounted for muscle glucose uptake. Other substrates, such as free fatty acids, muscle glycogen, and lactate are metabolized in active muscle cells. Nonetheless, glucose oxidation increases with exercise intensity, and glucose uptake increases fairly, in proportion to glycogen utilization with an increase in the intensity of exercise [31]. Third, the method used to define ROI. As FDG uptake was measured at an arbitrary site on the target muscle, it did not reflect the uptake of the entire muscle volume; therefore, further investigation of muscle metabolism using PET is required. Fourth, the sample size was limited due to concerns over radiation exposure. The effect size of the mean SUV of the peroneus brevis was 2.33 on the throwing side, which was greater than the expected level of 2.0; this was the basis for calculating the sample size before the investigation. Thus, the sample size of the pitcher and control groups ( $n=10$ each) in this investigation was appropriate. Despite these limitations, no study has employed PET-CT in 
the examination of the whole-body muscle metabolism (during pitching).

To the best of our knowledge, this is the first study to evaluate whole-body skeletal muscle metabolism during pitching. PET is a useful tool for measuring the skeletal muscle metabolism of the whole body in an environment that accurately replicates actual pitching and is, therefore, useful for assessing other dynamic activities. As EMG research can only measure specific parts of the body, it was necessary to integrate several different studies of various participants and methods to consider and devise a novel whole-body training method.

\section{Conclusions}

Whole-body muscle metabolism during pitching was investigated using PET-CT, and a significant increase in glucose metabolism was observed in muscle groups of the fingers and toes. In addition, asymmetric metabolism was observed in the thigh muscles. There was minimal accumulation of FDG in the rotator cuff and trunk muscles. This information would be useful in determining the training required for pitchers. These findings can be equally applied to other sporting activities that involve throwing.

\section{Abbreviations}

3D: 3-dimensional; CT: Computed tomography; EMG: Electromyography; FDG: ${ }^{18}$ F-fluorodeoxyglucose; MCP: Metacarpophalangeal; PET: Positron emission tomography; ROI: Regions of interest; SD: Standard deviation; SUV: Standardized uptake value

\section{Acknowledgments}

The authors thank the pitchers of the Kanazawa Gakuin University Baseball Club for participating in this study.

\section{Authors' contributions}

The study was designed by YT, JN, Al, TM, SK and HT. All authors contributed to the data collection and entry. Data were analyzed by YT. Data interpretation and manuscript preparation were undertaken by all authors. All authors read and approved the final manuscript.

\section{Funding}

Not applicable.

\section{Availability of data and materials}

The datasets generated during and/or analyzed during the current study are available from the corresponding author on reasonable request.

\section{Declarations}

Ethics approval and consent to participate

The study design was approved by the Ethics Committee of Kanazawa University Hospital (2251-1) and Kanazawa Advanced Medical Center (070209). All human experiments in this study followed the guidelines of the Declaration of Helsinki.

\section{Consent for publication}

Not applicable.

\section{Competing interests}

The authors declare that they have no competing interests.

\section{Author details}

'Department of Orthopaedic Surgery, Graduate School of Medical Science, Kanazawa University, 13-1 Takaramachi, Kanazawa 920-0934, Japan. ${ }^{2}$ Department of Orthopaedic Surgery, National Hospital Organization, Kanazawa Medical Center, Kanazawa, Japan. ${ }^{3}$ Department of Nuclear Medicine/Biotracer Medicine, Graduate School of Medical Science, Kanazawa University, Kanazawa, Japan. ${ }^{4}$ Kanazawa Advanced Medical Center, Kanazawa, Japan.

Received: 27 June 2020 Accepted: 25 February 2021

Published online: 06 March 2021

\section{References}

1. Werner SL, Gill TJ, Murray TA, Cook TD, Hawkins RJ. Relationships between throwing mechanics and shoulder distraction in professional baseball pitchers. Am J Sports Med. 2001;29:354-8.

2. Lyman S, Fleisig GS, Andrews JR, Osinski ED. Effect of pitch type, pitch count, and pitching mechanics on risk of elbow and shoulder pain in youth baseball pitchers. Am J Sports Med. 2002;30:463-8.

3. Chalmers PN, Wimmer MA, Verma NN, Cole BJ, Romeo AA, Cvetanovich GL, et al. The relationship between pitching mechanics and injury: a review of current concepts. Sports Health. 2017;9:216-21.

4. Campbell BM, Stodden DF, Nixon MK. Lower extremity muscle activation during baseball pitching. J Strength Cond Res. 2010;24:964-71.

5. Erickson BJ, Zaferiou A, Chalmers PN, Ruby D, Malloy P, Luchetti TJ, et al. Are the hamstrings from the drive leg or landing leg more active in baseball pitchers? An electromyographic study. J Shoulder Elbow Surg. 2017;26: 2010-6.

6. Escamilla RF, Andrews JR. Shoulder muscle recruitment patterns and related biomechanics during upper extremity sports. Sports Med. 2009:39:569-90.

7. Gowan ID, Jobe FW, Tibone JE, Perry J, Moynes DR. A comparative electromyographic analysis of the shoulder during pitching. Professional versus amateur pitchers. Am J Sports Med. 1987;15:586-90.

8. Jobe FW, Tibone JE, Perry J, Moynes D. An EMG analysis of the shoulder in throwing and pitching: a preliminary report. Am J Sports Med. 1983;11:3-5.

9. Sisto DJ, Jobe FW, Moynes DR, Antonelli DJ. An electromyographic analysis of the elbow in pitching. Am J Sports Med. 1987;15:260-3.

10. Yamanouchi T. EMG analysis of the lower extremities during pitching in high-school baseball. Kurume Med J. 1998;45:21-5.

11. Digiovine NM, Jobe FW, Pink M, Perry J. An electromyographic analysis of the upper extremity in pitching. J Shoulder Elbow Surg. 1992;1:15-25.

12. Jobe FW, Moynes DR, Tibone JE, Perry J. An EMG analysis of the shoulder in pitching: a second report. Am J Sports Med. 1984;12:218-20.

13. Erickson BJ, Sgori T, Chalmers PN, Vignona P, Lesniak M, Bush-Joseph CA, et al. The impact of fatigue on baseball pitching mechanics in adolescent male pitchers. Arthroscopy. 2016;32:762-71.

14. Watkins RG, Dennis S, Dillin WH, Schnebel B, Schneiderman G, Jobe F, et al. Dynamic EMG analysis of torque transfer in professional baseball pitchers. Spine. 1989;14:404-8.

15. Nakase J, Inaki A, Mochizuki T, Toratani T, Kosaka M, Ohashi Y, et al. Whole body muscle activity during the FIFA $11+$ program evaluated by positron emission tomography. PLoS One. 2013;8:e73898.

16. Takata Y, Nakase J, Inaki A, Mochizuki T, Numata H, Oshima T, et al. Changes in muscle activity after performing the FIFA 11+ programme part 2 for 4 weeks. J Sports Sci. 2016:34:2011-7.

17. Bakshi NK, Inclan PM, Kirsch JM, Bedi A, Agresta C, Freehill MT. Current workload recommendations in baseball pitchers: a systematic review. Am J Sports Med. 2020;48:229-41.

18. Bojsen-Møller J, Kalliokoski K, Seppanen M, Kjaer M, Magnusson SP. Lowintensity tensile loading increases intratendinous glucose uptake in the Achilles tendon. J Appl Physiol (1985). 2006;101:196-201.

19. Fujimoto T, Itoh M, Kumano H, Tashiro M, Ido T. Whole-body metabolic map with positron emission tomography of a man after running. Lancet. 1996;348:266

20. Ohnuma M, Sugita T, Kokubun S, Yamaguchi K, Rikimaru H. Muscle activity during a dash shown by 18F-fluorodexyglucose positron emission tomography. J Orthop Sci. 2006;11:42-5.

21. Tashiro M, Fujimoto T, Itoh M, Kubota K, Fujiwara T, Miyake M, et al. 18FFDG PET imaging of muscle activity in runners. J Nucl Med. 1999;40:70-6.

22. Rudroff T, Ketellhut NB, Kindred JH. Metabolic imaging in exercise physiology. J Appl Physiol (1985). 2018;124:497-503. 
23. Oi N, Iwaya T, Itoh M, Yamaguchi K, Tobimatsu Y, Fujimoto T. FDG-PET imaging of lower extremity muscular activity during level walking. J Orthop Sci. 2003;8:55-61.

24. Tai SJ, Liu RS, Kuo YC, Hsu CY, Chen CH. Glucose uptake patterns in exercised skeletal muscles of elite male long-distance and short-distance runners. Chin J Physiol. 2010;53:91-8.

25. Bojsen-Møller J, Losnegard T, Kemppainen J, Viljanen T, Kalliokoski KK, Hallén J. Muscle use during double poling evaluated by positron emission tomography. J Appl Physiol (1985). 2010;109:1895-903.

26. Hore J, Watts S, Leschuk M, MacDougall A. Control of finger grip forces in overarm throws made by skilled throwers. J Neurophysiol. 2001;86:2678-89.

27. Ketchum LD, Thompson D, Pocock G, Wallingford DA. Clinical study of forces generated by the intrinsic muscles of the index finger and the extrinsic flexor and extensor muscles of the hand. J Hand Surg Am. 1978;3:571-8.

28. Kinoshita H, Obata S, Nasu D, Kadota K, Matsuo T, Fleisig GS. Finger forces in fastball baseball pitching. Hum Mov Sci. 2017:54:172-81.

29. Shane TS, Shane JN, Bernard RB, Charles AB, Gregory PN, Anthony AR. The kinetic chain in overhand pitching: its potential role for performance enhancement and injury prevention. Sports Health. 2010;2:135-46.

30. Fleisig GS, Andrews JR, Dillman CJ, Escamilla RF. Kinetics of baseball pitching with implications about injury mechanisms. Am J Sports Med. 1995;23:233-9

31. Staron RS, Karapondo DL, Kraemer WJ, Fry AC, Gordon SE, Falkel JE, et al. Skeletal muscle adaptations during early phase of heavy-resistance training in men and women. J Appl Physiol (1985). 1994;76:1247-55.

\section{Publisher's Note}

Springer Nature remains neutral with regard to jurisdictional claims in published maps and institutional affiliations.

Ready to submit your research? Choose BMC and benefit from:

- fast, convenient online submission

- thorough peer review by experienced researchers in your field

- rapid publication on acceptance

- support for research data, including large and complex data types

- gold Open Access which fosters wider collaboration and increased citations

- maximum visibility for your research: over $100 \mathrm{M}$ website views per year

At $\mathrm{BMC}$, research is always in progress.

Learn more biomedcentral.com/submissions 